\title{
Light-Trapping of the European Corn Borer (Ostrinia nubilalis Hbn.) at Different Values of the Q-index Expressing the Different Intensities of Solar Flares
}

\author{
L. NOWINSZKY and J. PUSKÁS \\ Berzsenyi Dániel College, H-9701 Szombathely, P.O. Box 170, Hungary
}

\begin{abstract}
The paper deals with connections between solar flare activities and light-trap collection of insects. The authors have worked out the catch data of European corn borer (Ostrinia nubilalis Hbn.) adults, as published for the period of 1976-1997 by the Hungarian national light-trap network. The results proved that both the daily and annual catches were significantly modified by the Q-indexes, expressing the different lengths and intensities of the solar flares. On days with high Q-indexes relative to the ones of the average swarming periods, the number of catches are considerably lower. In those years when the Q-index is high, the average individual number and the number of caught moths are lower by $30 \%$ as compared to the average number of total cycles (11 years) and the average population density of a given biotope. On the contrary, in years with low Q-indexes an increase as high as $45 \%$ can be experienced in the number of individuals collected. Thus, by evaluating the light-trap catches a strong modifying effect of solar flares has to be considered.
\end{abstract}

Keywords: European corn borer, Ostrinia nubilalis, light-trap, solar flares, Q-index.

As part of global solar activity flares, eruptions can be observed in the active regions of the solar surface, that are accompanied by intensive X-ray, gamma- and corpuscular radiations, that reach also the Earth and establish an interaction with its outermost atmosphere, producing thus changes in its electromagnetic conditions (Smith and Smith, 1963).

The Sun eruptions, flares are short (lasting max. 10-20 minutes) shiny spots, temporal areas in the chromosphere of the Sun. Their observation is carried out conventionally in the red (656.3 nanometer wavelength light) of alpha line of hydrogen. At the time of intensive flares the corpuscular emission is thousandfold as compared to a quiet period of Sun. The corpuscles are mostly electrons flying into all directions (also toward the Earth) with a speed of $1500 \mathrm{~km}^{-1}$. These electrically charged corpuscles compose the socalled solar wind that - in contrast to the electromagnetic radiation that reaches the Earth in 8 and a half minutes - reaches our atmosphere in 26-28 hours. On their way to Earth, the flare particles have also to pass the interplanetary space. The magnetic field generated by the galactic cosmic radiation is moderating considerably the effects of flares exerted on the magnetosphere of Earth. Thus, not all flares cause changes in the physical conditions of the upper atmosphere. If, however these changes result from the above-mentioned reasons, the weather becomes temporarily altered and the magnetic field of the corpuscles modifies the undisturbed daily course of the terrestrial magnetic field. 
The flares are classified according to the size of their area as compared to the total solar surface. The flares of primary importance (1) do not reach 250 times the half of on millionth part of the total solar surface. If the flare takes 250-600 times this size, it receives an index number of 2 ; if greater 600 times than that, it has a significance of 3 . Because of their significant energy emissions, the cosmic influence of the flares No. 2 and 3 is the most considerable.

Most daily flare activities are characterised by most authors by index $Q$ that expresses the significance of flares also by their duration. Its calculation is made by the following formula:

$$
Q=(i \times t)
$$

where $i=$ flare intensity, $t=$ the time length of its existence

Earlier Örményi (1966) calculated and published the flare activity numbers based on similar theoretical principles ("Flare Activity Numbers") for the period of 1957-1965.

The solar activity also exerts influence on life phenomena. In the literature accessible to the authors, however, no publication can be found that would have dealt with the influence of flares on the collection of insects by light-traps. Earlier we have published our studies and demonstrated the influence of hydrogen alpha flares No. 2 and 3 (Tóth and Nowinszky, 1983) on light-trap catches.

\section{Material}

The Q-index daily data for the period 1976-1997 were provided by Dr. T. Atac (Bogazici University, Kandilli Observatory and Earthquake Research Institute, Istanbul) and by Dr. J. Verö (Research Institute of Geodesy and Geophysics, Hungarian Academy of Sciences, Sopron). Their help is here gratefully acknowledged.

The collection data of European corn borer (Ostrinia nubilalis Hbn.) were taken from the collection of the national light-trap network. The data of traps, operated by the Plant Protection Stations were used with the authorization of Dr. I. Eke (Ministry of Agriculture and Country Development), Dr. M. Tóth and Mrs. Gy. Mohai (Station of Plant Health and Soil Protection, Budapest). Many data were received from Professor Z. Mészáros (University of Horticulture and Food Industry, Budapest).

In course of the period studied: in 81 light-trap stations and 3114 nights 133419 moth individuals were collected in total. As more than one trap operated on each nights the authors disposed over 40336 observation data in preparing present paper.

\section{Methods}

From the collection data of the European corn borer (Ostrinia nubilalis Hbn.) relative catch (RC) data were calculated for each observation posts and days. The RC is the quotient of the number of individuals caught during a sampling time unit (1 night or 1 hour) per the average number of individuals of the same generation falling to the same 
time unit. In case of the expected average individual number the RC value is 1 . The introduction of RC enables us to carry out a joint evaluation of materials collected in different years and at different points.

At the values of Q-index showed considerable differences in course of the respective years, they were preferably expressed as percentages of the averages of swarming periods. In the first step we studied the influence of flare activities on the daily catches. To disclose the latter, the Q/Q average values were co-ordinated with the relative catch data of different observation posts for each day of the catch period. The Q/Q mean values have been contracted into groups (classes), then averaged within the classes the relative catches data pertaining to them.

In subsequent studies we have investigated, whether the constantly changing activities of flares had modified the numbers of trapped European corn borer (Ostrinia nubilalis Hbn.) individuals? For this study only the data of those observation posts could be used that had worked throughout at least one total solar activity cycle (1976-1986 or 1986-1997). The light-traps operated through both cycles only on two observation posts; additionally, in the first cycle 11 and in the second further 1 light-trap yielded data from a total period. The total catch of the individual years has been compared with the average catch number of 11 years, so essentially yearly relative catch data $(\mathrm{RCy})$ were calculated. These numbers were yearly compared to the average of Q-index, then in years with low (Q-index average 1-3), medium (Q-index average 3-10) and high (Q-index average higher than 10) flare activity the level of significance of relative catch data (RCy) deviation was tried by t-test.

\section{Results}

The connections between Q/Q averages and daily catches of European corn borer (Ostrinia nubilalis Hbn.) are presented in Table 1. The values of Q-index characteristic for each year are shown in Table 2. In the latter the yearly relative catch data (RCy) of the studied species are also presented.

\section{Discussion}

From the results several important consequences could be drawn. Between the $\mathrm{Q}$-indexes and maximal values of $\mathrm{Q} / \mathrm{Q}$ average a negative correlation $(\mathrm{r}=-0.715)$ could be found, that is significant at $99.9 \%$ level.

As proven by the data of Table 1, on those days when the value of Q/Q average surpasses 3, the catch number of European corn borer (Ostrinia nubilalis Hbn.) adults significantly regresses. Such high values occur mainly in years in which the Q-index averages are lower than 10 . This means that in those years when the flare activity is mostly moderate the rarely occurring high Q-indexes surpass considerably the average. In those years, however, where the average Q-index is higher than 10, the highest Q-index 
Table 1

Daily catches of European corn borer (Ostrinia nubilalis Hbn.) adults in 1976-1997 at different values of $\mathrm{Q} / \mathrm{Q}$ average

\begin{tabular}{ccc}
\hline Q/Q average & Average of relative catch & Number of data \\
1 & 2 & 3 \\
\hline 0.00 & 0.964 & 9541 \\
0.12 & 1.051 & 3144 \\
0.31 & 1.054 & 3626 \\
0.51 & 0.988 & 4112 \\
0.71 & 1.048 & 3673 \\
0.91 & 0.985 & 2695 \\
1.43 & 1.008 & 8488 \\
2.41 & 0.987 & 2666 \\
3.42 & 1.102 & 911 \\
4.97 & 0.870 & 825 \\
6.82 & 0.821 & 321 \\
12.21 & 0.783 & 334 \\
\hline
\end{tabular}

Notes: Correlation between $\mathrm{Q} / \mathrm{Q}$ average and the relative catch: $r=-0,8146$ (significant on levels higher than $99 \%$ )

values surpass much less the average. The unfavourable influence of flare activity, which exceeds considerably the average, is reflected in the decreasing number of daily light-trap catches. Because decreasing catches may be experienced on the first day of strong flare activity, we assume that the success of light-trapping is significantly and negatively influenced by the electromagnetic radiation arriving from the Sun. The decrease of catch numbers cannot be felt at Q/Q average values lower than 4 . To these values generally high Q-indexes are attached, at the same time the deviation from the average is lower.

One can suppose that the high flare activity generally decreases the number of caught individuals. The method used by us so far, i.e. the relative daily catches, calculated separately for each year per swarming period of the Lepidopteran is inadequate. By introducing and using the relative yearly catch (RCy) we succeeded to confirm the fact that in the case of high flare activity when the Q-index average was higher than 10 (the average catch number calculated for a period of 11 years, a Sun cycle and characteristic for the given observation point, does barely amount to $70 \%$ of the expected individual number collected. In years with low flare activity, however, the catch number exceeds the average at least by $44 \%$.

So, according to our results, changes in Sun flare activity considerably modify both the daily catch of European corn borer (Ostrinia nubilalis Hbn.) individuals and the number of individuals present. Both statements are important for plant protection prognostics. 
Table 2

Yearly relative catch (RCy) of European corn borer (Ostrinia nubilalis Hbn.) adults calculated as functions of Q-index yearly averages

\begin{tabular}{|c|c|c|c|c|c|}
\hline \multirow[t]{2}{*}{ Years } & \multicolumn{2}{|c|}{ Q-index } & \multirow{2}{*}{$\begin{array}{l}\mathrm{Q} / \mathrm{Q} \text { average } \\
\text { maximum }\end{array}$} & \multirow{2}{*}{$\begin{array}{c}\text { Number } \\
\text { of data }\end{array}$} & \multirow{2}{*}{$\begin{array}{c}\mathrm{RCy} \\
\text { average }\end{array}$} \\
\hline & maximum & average & & & \\
\hline 1986 & 5.36 & 0.406 & 13.20 & 13 & 2.079 \\
\hline 1996 & 11.48 & 0.478 & 24.02 & 3 & 1.052 \\
\hline 1976 & 4.98 & 0.632 & 7.88 & 13 & 1.294 \\
\hline 1997 & 12.28 & 0.642 & 19.13 & 3 & 0.443 \\
\hline 1995 & 12.00 & 0.734 & 16.35 & 3 & 1.423 \\
\hline 1994 & 12.08 & 0.973 & 12.42 & 3 & 0.734 \\
\hline 1985 & 21.31 & 1.459 & 14.61 & 13 & 1.467 \\
\hline 1987 & 16.21 & 2.917 & 5.56 & 3 & 1.289 \\
\hline$a$ & & 0.920 & & 54 & 1.440 \\
\hline 1977 & 32.27 & 3.059 & 10.55 & 13 & 0.788 \\
\hline 1983 & 37.29 & 3.360 & 4.03 & 13 & 1.163 \\
\hline 1984 & 42.09 & 3.573 & 11.78 & 13 & 0.863 \\
\hline 1988 & 36.90 & 6.880 & 4.37 & 3 & 1.280 \\
\hline 1992 & 33.56 & 8.453 & 4.88 & 3 & 1.642 \\
\hline 1993 & 36.92 & 9.243 & 10.99 & 3 & 0.634 \\
\hline$b$ & & 5.468 & & 48 & 0.984 \\
\hline 1978 & 65.23 & 10.719 & 6.09 & 13 & 0.653 \\
\hline 1979 & 56.40 & 12.151 & 3.46 & 13 & 0.536 \\
\hline 1980 & 53.38 & 15.350 & 3.39 & 13 & 0.330 \\
\hline 1981 & 51.29 & 15.757 & 3.12 & 13 & 0.737 \\
\hline 1982 & 112.31 & 16.313 & 5.77 & 13 & 1.088 \\
\hline 1989 & 55.10 & 16.461 & 3.33 & 3 & 0.766 \\
\hline 1990 & 43.40 & 16.556 & 3.57 & 3 & 0.620 \\
\hline 1991 & 68.22 & 19.464 & 4.44 & 3 & 1.115 \\
\hline$c$ & & 15.614 & & 74 & 0.689 \\
\hline
\end{tabular}

Notes: Levels of significance:

Between Q-index average and maximum of Q/Q average $99.9 \%$

Between $a-b$ lines of RCy values $95.0 \%$

Between $b-c$ lines of RCy values $95.0 \%$

Between $a-c$ lines of RCy values $99.9 \%$

\section{Literature}

Örményi, I. (1966): The relationship between geomagnetic activity and chromospheric H $\alpha$-flares. Acta Geodaet., Geophys. et Montanist. Acad. Sci. Hung. 1, 121-136.

Smith, H. J. and Smith, E. V. P. (1963): Solar flares. Macmillan Co., New York. 426.

Tóth, Gy. and Nowinszky, L. (1983): Influence of solar activity on the outbreaks and daily light-trap catches of Scotia segetum Schiff. Z. ang. Ent. 95, 83-92. 